\title{
LA-UR-20-24491
}

Approved for public release; distribution is unlimited.

Title:

Author(s): $\quad$ Lewellen, John W. IV

Intended for: Web

Issued:

2020-06-22

\section{SPACEBORNE ACCELERATORS PORTFOLIO}


Disclaimer:

Los Alamos National Laboratory, an affirmative action/equal opportunity employer, is operated by Triad National Security, LLC for the National Nuclear Security Administration of U.S. Department of Energy under contract 89233218CNA000001. By approving this article, the publisher recognizes that the U.S. Government retains nonexclusive, royalty-free license to publish or reproduce the published form of this contribution, or to allow others to do so, for U.S. Government purposes. Los Alamos National Laboratory requests that the publisher identify this article as work performed under the auspices of the U.S. Department of Energy. Los Alamos National Laboratory strongly supports academic freedom and a researcher's right to publish; as an institution, however, the Laboratory does not endorse the viewpoint of a publication or guarantee its technical correctness. 


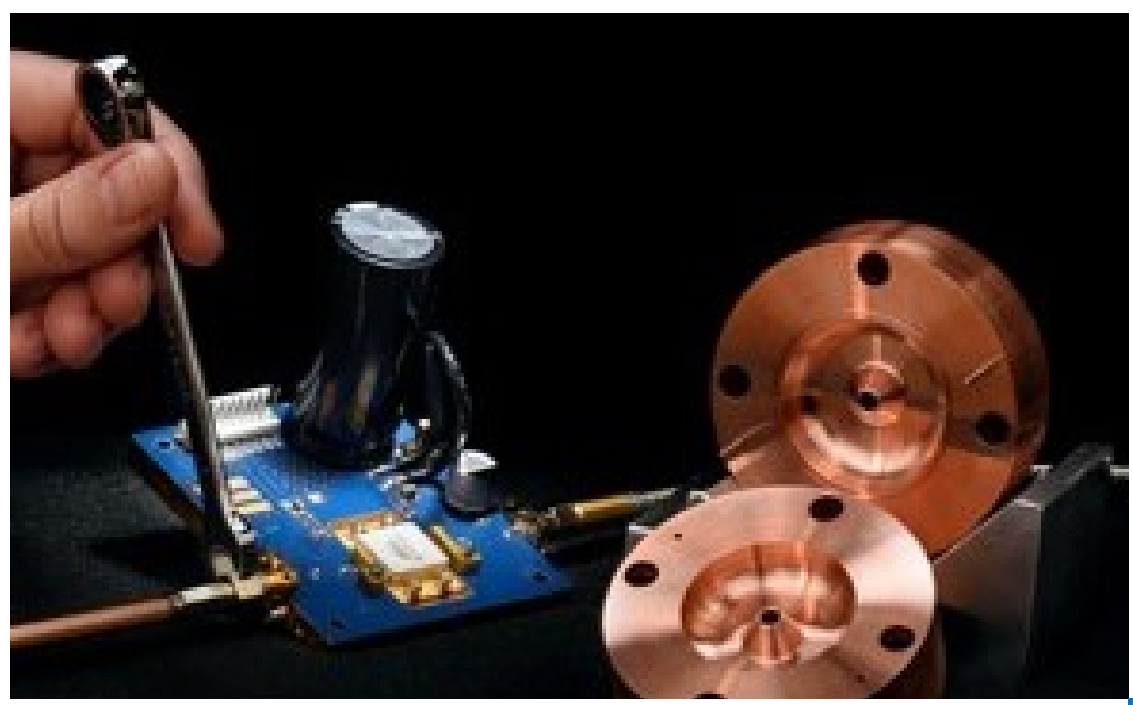

\section{SPACEBORNE ACCELERATORS PORTFOLIO}

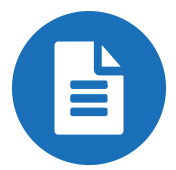

\section{SUMMARY}

MeV-range, satellite-mounted electron accelerators are needed for various space-related applications, such measuring the coupling between the magnetotail and ionosphere. The technologies and design approaches being developed for spaceborne accelerators emphasize high reliability, high average power, flexible operation, efficient power use, and operation from low-voltage DC power supplies. These same attributes are also relevant to industrial processing and inspection as well as medical applications.

\section{MARKET}

Many emerging applications for MeV-range electron accelerators, in medical, scientific, and industrial settings, have been identified by the DOE Basic Research Needs Workshop for Compact Electron Accelerators. The approaches developed for spaceborne accelerators can provide high-leverage improvements for terrestrial applications, particularly where high efficiency and high reliability are core requirements

\section{LosAlamos \\ NATIONAL LABORATORY \\ EST.1943 \\ Tech Snapshot Space}

Published: Jun 10, 2020

\section{BENEFITS}

A broad spectrum of accelerator and electrodynamics technology has been applied to a wide range of mission relevant programs and scientific disciplines, such as stockpile stewardship, material science, nuclear physics, national security and radioisotope production. As a key enabling technology, our demonstrated capabilities in the construction and operation of accelerators has created a significant infrastructure providing the depth required to enable our R\&D activities for spaceborne accelerators.

- Single-cell cavity drive provides highly redundant operation and helps eliminate single-points-of-failure.

- Supplies high average power - up to $10 \%$ duty factor operation.

- Operates from 50 - 100 VDC - eliminates high voltage, can run off battery packs.

- Modular design facilitates ready scaling.

- Adapts to a variety of beam sources, depending on application (e.g. thermionic or photocathode, DC or RF injector)

\section{CONTACT}

Ross Muenchausen

rossm@lanl.gov

505-664-0558 

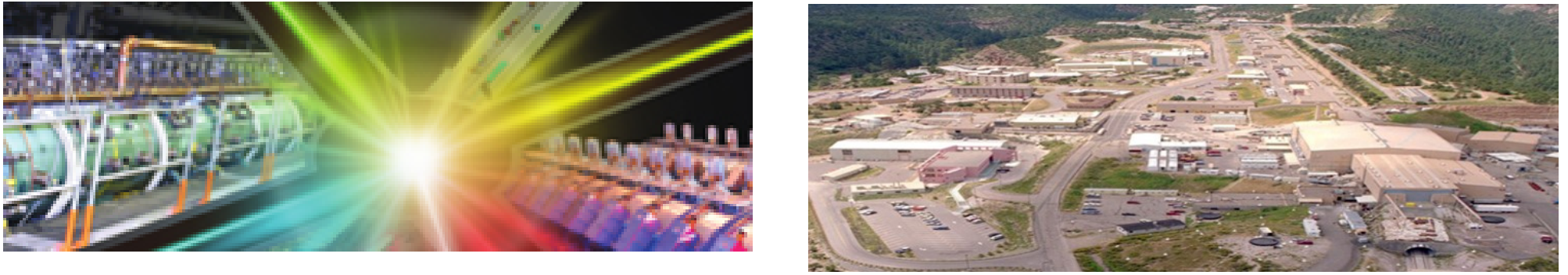

a. WHY WE ARE BUILDING SPACEBORNE ACCELERATORS PORTFOLIO

National security, scientific, industrial and medical problems require accelerator science, technology, and engineering for compact, MeVrange, high-reliability electron beam generation. Direct applications of electron beams effect aerospace, automotive, electrical, environmental and plastics industrial processing. Indirect applications of electron beams, to generate secondary particles or radiation, such as free electron lasers are also important.

\section{WHAT'S BEHIND OUR TECHNOLOGY}

High average power proton linear accelerator (linac) technology is an area where Los Alamos demonstrates world leadership. Conventional radio-frequency driven accelerators have been the workhorse of nuclear science and material science, as demonstrated at Los Alamos where an 800-MeV high-average-power proton linac generates both primary proton and secondary neutron particle beams for mission critical applications. Technological advances with linacs opened up two entirely new applications: accelerator-driven production of tritium and accelerator-driven systems for energy production.

\section{OUR COMPETITIVE ADVANTAGES}

Los Alamos National Laboratory maintains world-class, reliable accelerator operations in support of a broad portfolio of fundamental and applied R\&D. Ensuring long-term continuing operations and reestablishing high-average-power operations will enable even broader applications such as: high-energy isotope production for medical and weapons applications, intensity-frontier nuclear and particle physics, high-power spallation target testing, fission/fusion materials testing, and alternative fuels irradiation for reactor conversion.

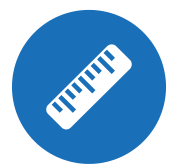

\section{OUR TECHNOLOGY STATUS}

Los Alamos scientists and engineers have reported on the basic principles, formulated the technology concepts and demonstrated various proofs-of-concept (TRL 3). Commercial partners are sought to further development toward full system level implementation (TRL 6). Patent licensing and/ or collaborative agreements can be tailored to facilitate commercial development

\section{PUBLICATIONS AND IP}

US Patent 10,211,505: Sideline radio-frequency power coupler

US Patent 10,568,196B1: Compact, high-efficiency accelerators driven by low-voltage solid-state amplifiers.

"Tutorial on X-Ray Free-Electron Lasers" , Carlsten, B. E.,IEEE TRANS. PLASMA SCI. ; 46, iss.6, , p.1900-1912, JUN 2018.

"Space-Borne Electron Accelerator Design", Lewellen, J. W., Buechler, C; Carlsten, B. E. et al. FRONTIERS IN ASTRONOMY AND SPACE SCIENCES ; 6, p.35, MAY 152019.

"Synergies with NNSA applications" ; Carlsten, Bruce Eric; GARD Accelerator and Beam Physics Workshop 2 ; Los Alamos, New Mexico, United States LA-UR-20-23378 MAY 52020. 